\title{
The predictive value of enhanced liver fibrosis (ELF) score for the patient with hepatocellular carcinoma after liver resection
}

\author{
Munseok CHOI${ }^{1}$, Alshandeer Marei HUSSAIN ${ }^{2}$, Dai Hoon HAN ${ }^{* 1}, \mathrm{Gi}_{\mathrm{H}}$ Hong $\mathrm{CHOI}^{1}$, Jin Sub $\mathrm{CHOI}^{1}$ \\ 'Division of Hepatobiliary and Pancreatic Surgery, Department of Surgery, Yonsei University College of Medicine, Seoul, Korea \\ ${ }^{2}$ Department of Surgery, King Khalid University, Abha, Saudi Arabia
}

Introduction: The enhanced liver fibrosis (ELF) score is and extracellular matrix marker set consisting of tissue inhibitor of metalloproteinases 1 (TIMP-1), amino-terminal propeptide of type III procollagen (PIIINP) and hyaluronic acid (HA) having good correlations with fibrosis stages in chronic liver disease. This study aimed to figure out the predictive value of ELF score for the hepatocellular carcinoma (HCC) patients having liver resection.

Methods: From April 2015 to July 2020, preoperative ELF score was collected for 127 patients with HCC having curative liver resection. The patients were grouped according to ELF score as 11 and perioperative outcome was compared between two groups: high ELF (HELF, $\mathrm{n}=22$ ) vs. low ELF (LELF, $\mathrm{n}=105$ ). Perioperative prognostic factors were analyzed for disease-free survival after liver resection.

Results: HELF had significantly higher liver stiffness by transient elastography ( $8.7 \pm 4.0$ vs. $23.4 \pm 16.4$ Kpa; $p=0.001)$, and higher perioperative transfusion $(3.8 \%$ vs. $22.7 \% ; p=0.007)$. Disease-free survival rates of HELF was significantly poor comparing LELF group. Tumor size more than $5 \mathrm{~cm}(\mathrm{HR}, 3.559$; range, $1.487-8.518 ; p=0.004)$, and HELF $(\mathrm{HR}, 2.745 ;$ range, $1.063-7.083 ; p=0037)$ were the significantly poor risk factors for disease-free survival on multivariate analysis.

Conclusions: Higher ELF score may be correlated with poor liver condition correlating liver fibrosis. Moreover, ELF score was the significant prognostic factor for disease-free survival for the patient having HCC after liver resection. 